\title{
Reclassification of Acinetobacter grimontii Carr et al. 2003 as a later synonym of Acinetobacter junii Bouvet and Grimont 1986
}

Correspondence

Mario Vaneechoutte

Mario.Vaneechoutte@UGent.be

\author{
Mario Vaneechoutte, ${ }^{1}$ Thierry De Baere, ${ }^{1}$ Alexandr Nemec, ${ }^{2}$ \\ Martin Musílek, ${ }^{2}$ Tanny J. K. van der Reijden ${ }^{3}$ and Lenie Dijkshoorn ${ }^{3}$ \\ ${ }^{1}$ Department of Clinical Chemistry, Microbiology and Immunology, University Hospital, Blok A, \\ B-9000 Gent, Belgium \\ ${ }^{2}$ Centre of Epidemiology and Microbiology, National Institute of Public Health, Šrobárova 48, 100 \\ 42 Prague, Czech Republic \\ ${ }^{3}$ Department of Infectious Diseases, Leiden University Medical Center C5-P, PO Box 9600, 2300 \\ RC Leiden, The Netherlands
}

\begin{abstract}
Using tDNA-PCR, the type strain CCM $7198^{\top}\left(\leftarrow\right.$ CIP $\left.107470^{\top} \leftarrow 17 \mathrm{A04}{ }^{\top}\right)$ of Acinetobacter grimontii was found to be indistinguishable from Acinetobacter junii strains. Therefore, the phenotypic properties, amplified fragment length polymorphism (AFLP) patterns and 16S rRNA and $r p o B$ gene sequences of the type strain of $A$. grimontii (CCM $\left.7198^{\top}\right)$ were determined. We found that the strain used L-arginine and L-glutamate, in contrast to the original description and in accordance with the phenotypic properties of $A$. junii. By AFLP analysis, A. grimontii CCM $7198^{\top}$ clustered at $50.2 \%$ with a set of $A$. junii strains previously identified by DNA-DNA hybridization, which is in accordance with the previously established intraspecies values of this technique. Sequence similarity of the 16S rRNA gene between the type strains of the two species was found to be $99.9 \%$. Finally, DNA-DNA relatedness between the type strains of $A$. junii and $A$. grimontii was redetermined and was found to be $85 \%$. These findings were corroborated for a second representative of the $A$. grimontii type strain, DSM $14968^{\top}$. These data confirm that Acinetobacter grimontii is a later heterotypic synonym of Acinetobacter junii.
\end{abstract}

Carr et al. (2003) described Acinetobacter grimontii as one of seven novel species from activated sludge from Australia on the basis of $16 \mathrm{~S}$ rRNA gene sequence analysis, phenotypic characterization and DNA-DNA hybridization. The type strain of $A$. grimontii $\left(17 \mathrm{~A} 04^{\mathrm{T}}\right)$ was deposited at two public culture collections, the Collection Institut Pasteur, Paris, France (CIP $107470^{\mathrm{T}}$ ) and the Deutsche Sammlung von Mikroorganismen und Zellkulturen, Braunschweig, Germany (DSMZ; DSM 14968 ${ }^{\mathrm{T}}$ ). A subculture of CIP $108470^{\mathrm{T}}$ was subsequently obtained by the Czech Collection of Microorganisms, Prague, Czech Republic, and designated CCM $7198^{\mathrm{T}}$.

When comparing the tDNA-PCR pattern (Baele et al., 2000, 2001; Vaneechoutte et al., 1998) of A. grimontii CCM $7198^{\mathrm{T}}$ with those of other Acinetobacter strains, we found the strain to be indistinguishable from Acinetobacter junii strains (data not shown). Later, we also found A. grimontii

Abbreviation: AFLP, amplified fragment length polymorphism.

The GenBank/EMBL/DDBJ accession numbers for the 16S rRNA gene sequences of $A$. junii ATCC $17908^{\top}, A$. junii SH215.SHVI (=ACI289) and A. grimontii CCM $7198^{\top}$ are respectively AM410704-AM410706.
DSM $14968^{\mathrm{T}}$ to be indistinguishable from $A$. junii by tDNA-PCR (not shown).

To reassess the taxonomic relationships of $A$. grimontii and A. junii, phenotypic analysis and amplified fragment length polymorphism (AFLP) fingerprinting were carried out for a selection of $A$. junii strains and both the CCM and DSMZ representatives of the $A$. grimontii type strain, the $16 \mathrm{~S}$ rRNA gene sequence was determined for both representatives of the type strain, the $r p o B$ gene sequence was determined for A. grimontii CCM $7198^{\mathrm{T}}$ and DNA-DNA hybridization was performed between A. grimontii CCM $7198^{\mathrm{T}}$ and A. junii LMG $998^{\mathrm{T}}$.

Phenotypic analysis for A. grimontii CCM $7198^{\mathrm{T}}$ and DSM $14968^{\mathrm{T}}$ and four A. junii strains which had previously been assigned to the species by DNA-DNA hybridization (CCM $2376^{\mathrm{T}}$, RUH 204, RUH 2229, RUH 2230) was carried out as described by Nemec et al. (2001). None of the six strains produced acid from D-glucose or hydrolysed gelatinase. Two strains (RUH 2229, RUH 2230) showed haemolytic activity on sheep-blood agar at 30 or $37{ }^{\circ} \mathrm{C}$, while the others were non-haemolytic. When inoculated onto brain heart infusion broth (Oxoid), all strains showed clear 
growth after 1 day at $37{ }^{\circ} \mathrm{C}$, while showing weak or doubtful growth at $41{ }^{\circ} \mathrm{C}$ and no growth at $44{ }^{\circ} \mathrm{C}$ within 2 days of incubation. In assimilation tests, modified from the method of Bouvet \& Grimont (1986), all strains utilized DL-lactate, L-arginine, L-glutamate and acetate with clearly positive results after 2 days of incubation, whereas none of the strains grew on trans-aconitate, glutarate, azelate, $\beta$-alanine, malonate, histamine, L-phenylalanine, phenylacetate, laevulinate, citraconate, 4-hydroxybenzoate, L-tartrate, L-ornithine, 2,3-butanediol, L-arabinose, D-glucose, D-gluconate, gentisate, tryptamine, trigonelline, tricarballylate, putrescine or adipate within 10 days. With the exceptions indicated in parentheses, all strains utilized Simmons' citrate (RUH 2229), L-histidine (RUH 2230), ethanol (RUH 2230) and 4-aminobutyrate (RUH 2229, RUH 2230), while only one strain (CCM $2376^{\mathrm{T}}$ ) grew on L-aspartate. There was no difference in the phenotypic properties of A. grimontii CCM $7198^{\mathrm{T}}$ and A. grimontii DSM $14968^{\mathrm{T}}$. Whereas Carr et al. (2003) reported that A. grimontii could be differentiated from $A$. junii by its inability to assimilate L-arginine or L-glutamate, we found that A. grimontii strains CCM $7198^{\mathrm{T}}$ and DSM $14968^{\mathrm{T}}$ clearly grew on both L-arginine and L-glutamate within 2 days of incubation. Thus, the phenotypic properties of A. grimontii reported here are fully consistent with those of A. junii.

AFLP analysis was carried out as described previously (Nemec et al., 2001). Briefly, DNA was digested simultaneously with EcoRI and MseI and with simultaneous adaptor ligation. PCR was carried out with a Cy5-labelled $E c o R I+A$ primer and an $M s e I+C$ primer. Amplified fragments were separated with the ALF Express system (Amersham Biosciences). Cluster analysis of profiles was done with the BioNumerics software release 4.5 (Applied Maths) with Pearson's product moment coefficient $(r)$ as a measure of similarity and the unweighted pair group average linkage method for clustering. Profiles were compared to a library of reference strains of all described species. Generally, the grouping of strains according to their AFLP similarity at $\sim 50 \%$ corresponds to the grouping obtained by employing a DNA-DNA hybridization cut-off of $70 \%$ (Nemec et al., 2001). Cluster analysis of AFLP profiles of A. grimontii CCM $7198^{\mathrm{T}}$ with those of reference strains of all described named and unnamed Acinetobacter species, all identified by DNA-DNA hybridization, showed that A. grimontii falls within the A. junii cluster (Fig. 1). This cluster, which is delineated at a clustering level of $50.2 \%$, was well separated from all other species, with Acinetobacter haemolyticus being closest, at $19.9 \%$. AFLP analysis showed a same-run similarity of $96.5 \%$ for the two representatives of the A. grimontii type strain (CCM $7198^{\mathrm{T}}$ and DSM $14968^{\mathrm{T}}$ ), which is above the strain delineation level of $90 \%$ determined previously (van den Broek et al., 2006) (data not shown).

From the publication of Carr et al. (2003), it could be observed that the $16 \mathrm{~S}$ rRNA gene sequence of $A$. grimontii $17 \mathrm{~A} 04^{\mathrm{T}}$ as published in their study showed highest

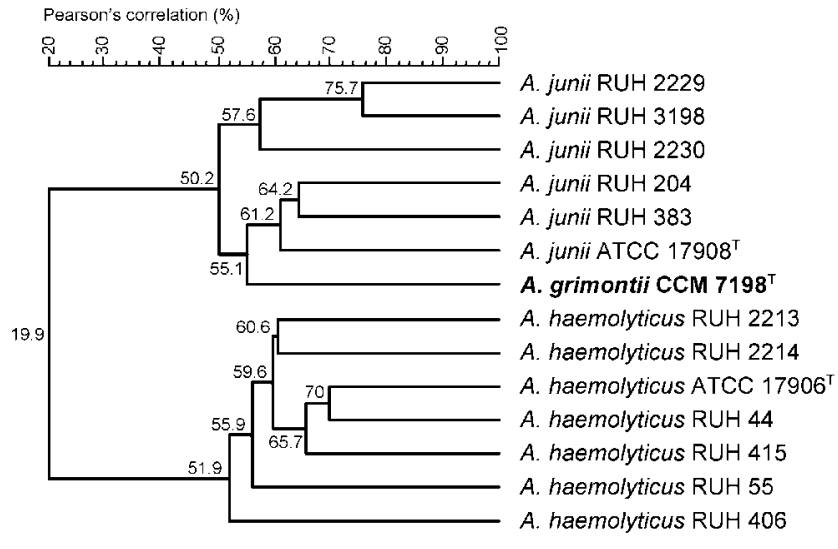

Fig. 1. Dendrogram of AFLP fingerprints obtained from the type strains of $A$. junii and $A$. grimontii and from $A$. junii and A. haemolyticus strains already present in the Leiden University Medical Centre (LUMC) database. All strains were previously identified by DNA-DNA hybridization. Numbers on branches indicate similarity values. $\mathrm{LUH}$ and $\mathrm{RUH}$ are designations of strains of the LUMC strain collection.

similarity to that of the type strain of A. junii, i.e. $99 \%$, with 7 mismatches and 20 gaps over the compared length of $1310 \mathrm{bp}$. Here, we redetermined the 16S rRNA gene sequence of the A. grimontii type strain, as obtained from both the CCM and DSMZ. The 16S rRNA gene sequence of strain CCM $7198^{\mathrm{T}}$ was $100 \%$ identical to that of strain DSM $14968^{\mathrm{T}}$. The A. grimontii type strain sequence was found to be even more similar (99.9\%) to that of the type strain of A. junii (Fig. 2) than was reported by Carr et al. (2003). The only differences between the two sequences are an ambiguity in the sequence of the A. junii type strain, which was determined to be adenine (A) in our sequence, and the presence of two different gaps, one in each sequence.

Finally, the synonymy of the two species names is corroborated by an independent source. La Scola et al. (2006), reporting on $r p o B$ gene sequencing as an approach with high discriminatory power, found that the complete rpoB gene sequences ( $4089 \mathrm{bp}$ ) of the type strains of $A$. junii and A. grimontii showed $99 \%$ similarity whereas, for all other species pairs except for A. baylyi and Acinetobacter genomic species 11 , less than $95 \%$ similarity was observed in pairwise comparisons. In addition, $r p o B$ sequences were determined in this study for A. junii CCM $2376^{\mathrm{T}}$ and A. grimontii CCM $7198^{\mathrm{T}}$ for both zones 1 and 2 (according to La Scola et al., 2006). Firstly, our sequencing results, obtained for the CCM type strains for both species, were $100 \%$ identical to those published by La Scola et al. (2006), obtained for the CIP type strains, for both strains and zones, confirming that the A. grimontii type strain held by the CCM was indeed obtained from the CIP. Secondly, we established $99 \%$ identity between $A$. junii CCM $2376^{\mathrm{T}}$ and A. grimontii CCM $7198^{\mathrm{T}}$ for $r p o B$ zone 1 and $97 \%$ identity 


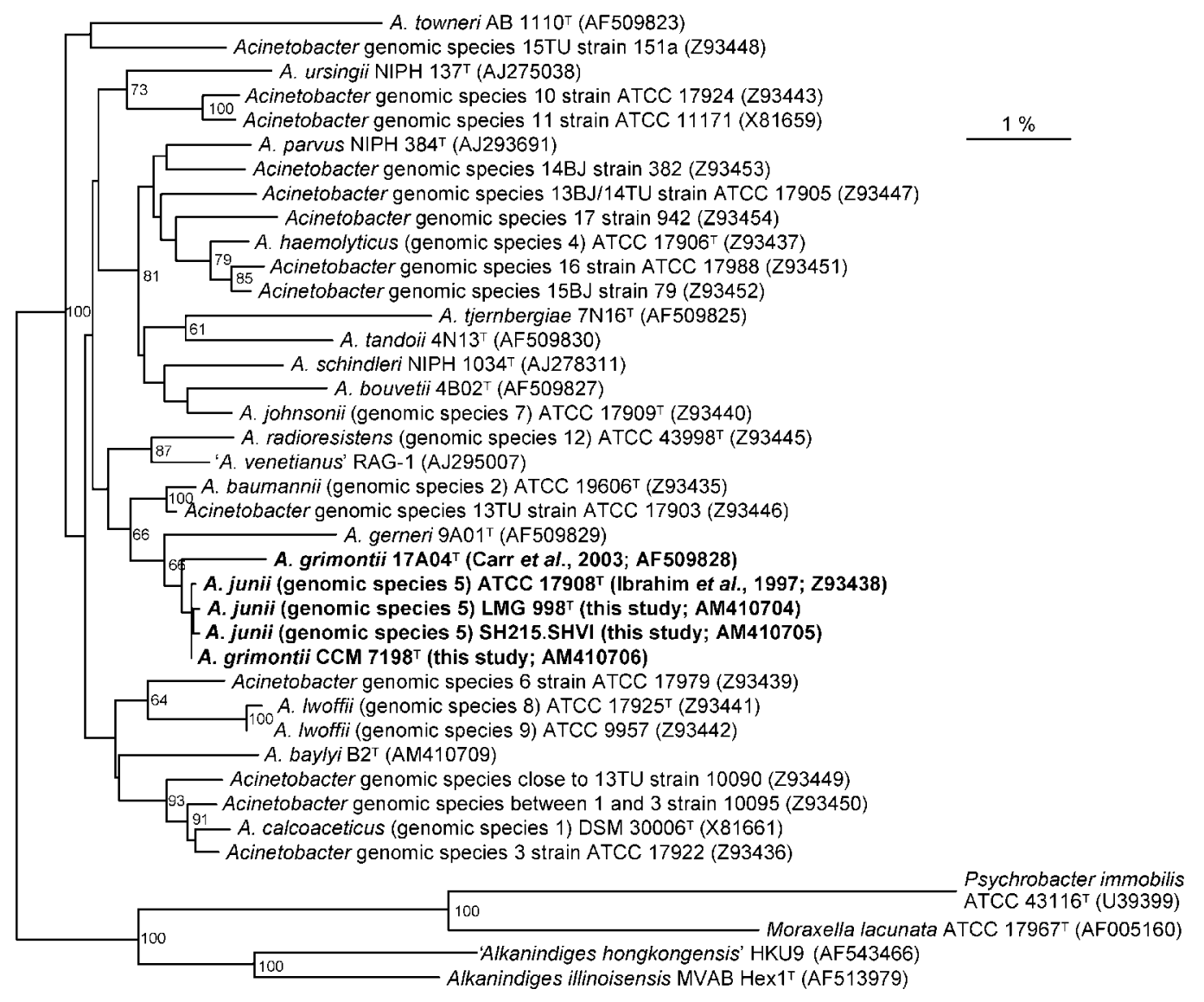

Fig. 2. Rooted tree showing phylogenetic relationships within the genus Acinetobacter on the basis of a region of the $16 \mathrm{~S}$ rRNA gene sequence corresponding to nucleotides 110-1443 of the gene from Escherichia coli ATCC $11775^{\top}$ (GenBank accession no. X80725). Cluster analysis was performed using Genebase (Applied Maths) and the tree was constructed on the basis of the neighbour-joining method using members of the closest related genus Alkanindiges as an outgroup. Bar, $1 \%$ sequence diversity. Numbers at nodes indicate bootstrap values. NIPH, National Institute of Public Health, Prague, Czech Republic. Acinetobacter sequences not determined in this study were reported by Rainey et al. (1994), Ibrahim et al. (1997), Vaneechoutte et al. (1999), Nemec et al. (2001, 2003) and Carr et al. (2003).

for $r p o B$ zone 2, corroborating the synonymy of A. grimontii and A. junii.

Carr et al. (2003) reported that the DNA-DNA relatedness between the type strains of $A$. grimontii and $A$. junii was $63 \%$, which was the highest of all interspecies values reported in their study. In our study, DNA-DNA hybridization between A. junii LMG $998^{\mathrm{T}}$ and A. grimontii CCM $7198^{\mathrm{T}}$ was carried out by the Culture Collection of the LMG (Gent, Belgium). Total DNA was prepared according to a modification of the method described by Wilson (1987), while hybridizations were performed at $37{ }^{\circ} \mathrm{C}$ according to a modification of the method described by Ezaki et al. (1989). A DNA-DNA relatedness value of $85 \%$ (mean of at least four hybridizations) was obtained, with a maximum difference of 21 units between the reciprocal values. With this technique, the mean standard deviation is 14 units and deviations up to 20-25 units are still acceptable (Goris et al., 1998). In conclusion, the value of $85 \%$ is clearly above $70 \%$, the generally accepted limit for species delineation (Wayne et al., 1987), and indicates that the two strains belong to the same species.

All data presented here, as well as results published independently by La Scola et al. (2006), clearly indicate the synonymy of $A$. grimontii and A. junii. The reclassification of Acinetobacter grimontii Carr et al. 2003 as a later heterotypic synonym of Acinetobacter junii Bouvet and Grimont 1986 is therefore proposed in accordance with Rule 24a of the Bacteriological Code (Lapage et al., 1992).

\section{Acknowledgements}

T.D. B. is indebted to the FWO (Fonds voor Wetenschappelijk Onderzoek - Vlaanderen) for a post-doctoral fellowship. 


\section{References}

Baele, M., Baele, P., Vaneechoutte, M., Storms, V., Butaye, P., Devriese, L. A., Verschraegen, G., Gillis, M. \& Haesebrouck, F. (2000). Application of tDNA-PCR for the identification of Enterococcus species. J Clin Microbiol 38, 4201-4207.

Baele, M., Storms, V., Haesebrouck, F., Devriese, L. A., Gillis, M., Verschraegen, G., De Baere, T. \& Vaneechoutte, M. (2001). Application and evaluation of the interlaboratory reproducibility of tRNA intergenic length polymorphism analysis (tDNA-PCR) for identification of species of the genus Streptococcus. J Clin Microbiol 39, 1436-1442.

Bouvet, P. J. M. \& Grimont, P. A. D. (1986). Taxonomy of the genus Acinetobacter with the recognition of Acinetobacter baumannii sp. nov., Acinetobacter haemolyticus sp. nov., Acinetobacter johnsonii sp. nov., and Acinetobacter junii sp. nov. and emended descriptions of Acinetobacter calcoaceticus and Acinetobacter lwoffii. Int J Syst Bacteriol 36, 228-240.

Carr, E. L., Kämpfer, P., Patel, B. K. C., Gürtler, V. \& Seviour, R. J. (2003). Seven novel species of Acinetobacter isolated from activated sludge. Int J Syst Evol Microbiol 53, 953-963.

Ezaki, T., Hashimoto, Y. \& Yabuuchi, E. (1989). Fluorometric deoxyribonucleic acid-deoxyribonucleic acid hybridization in microdilution wells as an alternative to membrane filter hybridization in which radioisotopes are used to determine genetic relatedness among bacterial strains. Int J Syst Bacteriol 39, 224-229.

Goris, J., Suzuki, K., De Vos, P., Nakase, T. \& Kersters, K. (1998). Evaluation of a microplate DNA-DNA hybridization method compared with the initial renaturation method. Can J Microbiol 44, 1148-1153.

Ibrahim, A., Gerner-Smidt, P. \& Liesack, W. (1997). Phylogenetic relationship of the twenty-one DNA groups of the genus Acinetobacter as revealed by $16 \mathrm{~S}$ ribosomal DNA sequence analysis. Int $J$ Syst Bacteriol 47, 837-841.

La Scola, B., Gundi, V. A. K. B., Khamis, A. \& Raoult, D. (2006). Sequencing of the $r p o B$ gene and flanking spacers for molecular identification of Acinetobacter species. J Clin Microbiol 44, 827-832.

Lapage, S. P., Sneath, P. H. A., Lessel, E. F., Skerman, V. B. D., Seeliger, H. P. R. \& Clark, W. A. (editors) (1992). International Code of
Nomenclature of Bacteria (1990 Revision). Bacteriological Code. Washington, DC: American Society for Microbiology.

Nemec, A., De Baere, T., Tjernberg, I., Vaneechoutte, M., van der Reijden, T. J. K. \& Dijkshoorn, L. (2001). Acinetobacter ursingii sp. nov. and Acinetobacter schindleri sp. nov., isolated from human clinical specimens. Int J Syst Evol Microbiol 51, 1891-1899.

Nemec, A., Dijkshoorn, L., Cleenwerck, I., De Baere, T., Janssens, D., van der Reijden, T. J. K., Jezek, P. \& Vaneechoutte, M. (2003). Acinetobacter parvus sp. nov., a small-colony-forming species isolated from human specimens. Int J Syst Evol Microbiol 53, 1563-1567.

Rainey, F. A., Lang, E. \& Stackebrandt, E. (1994). The phylogenetic structure of the genus Acinetobacter. FEMS Microbiol Lett 124, 349-354.

van den Broek, P. J., Arends, J., Bernards, A. T., De Brauwer, E., Mascini, E. M., van der Reijden, T. J., Spanjaard, L., Thewessen, E. A., van der Zee, A. \& other authors (2006). Epidemiology of multiple Acinetobacter outbreaks in The Netherlands during the period 19992001. Clin Microbiol Infect 12, 837-843.

Vaneechoutte, M., Boerlin, P., Tichy, H.-V., Bannerman, E., Jäger, B. \& Bille, J. (1998). Comparison of PCR-based DNA fingerprinting techniques for the identification of Listeria species and their use for atypical Listeria isolates. Int J Syst Bacteriol 48, 127-139.

Vaneechoutte, M., Tjernberg, I., Baldi, F., Pepi, M., Fani, R., Sullivan, E. R., van der Toorn, J. \& Dijkshoorn, L. (1999). Oil-degrading Acinetobacter strain RAG-1 and strains described as 'Acinetobacter venetianus sp. nov.' belong to the same genomic species. Res Microbiol 150, 69-73.

Wayne, L. G., Brenner, D. J., Colwell, R. R., Grimont, P. A. D., Kandler, O., Krichevsky, M. I., Moore, L. H., Moore, W. E. C., Murray, R. G. E. \& other authors (1987). International Committee on Systematic Bacteriology. Report of the ad hoc committee on reconciliation of approaches to bacterial systematics. Int J Syst Bacteriol 37, 463-464.

Wilson, K. (1987). Preparation of genomic DNA from bacteria. In Current Protocols in Molecular Biology, pp. 2.4.1-2.4.5. Edited by F. M. Ausubel, R. Brent, R. E. Kingston, D. D. Moore, J. G. Seidman, J. A. Smith \& K. Struhl. New York: Greene Publishing and Wiley-Interscience. 